\title{
Liver health in adults with Fontan circulation: A multicenter cross-sectional study
}

Fred M. Wu, MD, ${ }^{\mathrm{a}, \mathrm{b}}$ Brian Kogon, MD, ${ }^{\mathrm{c}}$ Michael G. Earing, MD, ${ }^{\mathrm{d}}$ Jamil A. Aboulhosn, MD, Craig S. Broberg, MD, ${ }^{\mathrm{f}}$ Anitha S. John, MD, ${ }^{\mathrm{g}}$ Amy Harmon, BA, ${ }^{\mathrm{a}}$ Nisha I. Sainani, MD, ${ }^{\mathrm{h}}$ Andrew J. Hill, MD, ${ }^{\text {h }}$ Robert D. Odze, MD ${ }^{i}$ Melanie E. Johncilla, MD, ${ }^{i}$ Chinweike Ukomadu, MD, PhD, ${ }^{j}$ Kimberlee Gauvreau, ScD, ${ }^{\mathrm{a}}$ Anne Marie Valente, MD, ${ }^{\mathrm{a}, \mathrm{b}}$ and Michael J. Landzberg, MD, ${ }^{\mathrm{a}, \mathrm{b}}$ for the Alliance for Adult Research in Congenital Cardiology (AARCC) Investigators

\section{ABSTRACT}

Objectives: Liver disease is an important contributor to morbidity and mortality in patients after Fontan surgery. There has been no large-scale survey of liver health in this population. We sought to explore the prevalence and predictors of liver disease in a multicenter cohort of adults with Fontan physiology.

Methods: Subjects were recruited from 6 adult congenital heart centers. Demographics; clinical history; and laboratory, imaging, and histopathology data were obtained.

Results: Of 241 subjects (median age 25.8 years [11.8-59.4], median time since Fontan 20.3 years [5.4-34.5]), more than $94 \%$ of those who underwent testing (208 of 221) had at least 1 abnormal liver-related finding. All hepatic imaging $(\mathrm{n}=54)$ and liver histology $(\mathrm{n}=68)$ was abnormal. Subjects with abnormal laboratory values had higher sinusoidal fibrosis stage ( 2 vs $1, P=.007)$ and higher portal fibrosis stage ( 3 vs $1, P=.003$ ) compared with those with all normal values. Low albumin correlated with lower sinusoidal fibrosis stage ( 1 vs $2 ; P=.02)$ and portal fibrosis stage ( 0 vs $3, P=.002$ ); no other liver studies correlated with fibrosis. Regenerative nodules were seen on $33 \%$ of histology specimens.

Conclusions: Regardless of modality, findings of liver disease are common among adults with Fontan circulation, even those appearing clinically well. Cirrhosis is present in up to one-third of subjects. Correlations between hepatic fibrosis stage and clinical history or findings on noninvasive testing are few. Further research is needed to identify patients at risk for more severe liver disease and to determine the best methods for assessing liver health in this population. (J Thorac Cardiovasc Surg 2017;153:656-64)



Hepatic fibrosis, present in nearly all cases, ranges from mild (A) to cirrhotic (D).

\section{Central Message}

Evidence of liver disease can be found in nearly all patients with Fontan circulation, but its clinical significance remains incompletely understood.

\section{Perspective}

As more people with Fontan circulation survive into adulthood, dysfunction of other organ systems has become apparent. In this first multicenter study of adults with Fontan circulation, evidence of liver disease is seen almost universally, but few correlations with clinical features are found. Clinicians caring for patients with Fontan circulation should include assessment of the liver as part of routine surveillance.

See Editorial Commentary page 665.
The development of the Fontan procedure was a major milestone in the management of patients with functionally univentricular hearts. ${ }^{1}$ The rerouting of systemic venous blood directly into the pulmonary circulation results in near normalization of systemic oxygen saturation and

\footnotetext{
From the ${ }^{a}$ Department of Cardiology, Boston Children's Hospital, and Divisions of ${ }^{\mathrm{b}}$ Cardiology and ${ }^{\mathrm{j}}$ Gastroenterology, and Departments of ${ }^{\mathrm{h}}$ Radiology and ${ }^{i}$ Pathology, Brigham and Women's Hospital, Harvard Medical School, Boston, Mass; ${ }^{\mathrm{c} D i v i s i o n}$ of Cardiothoracic Surgery, Emory University School of Medicine and Children's Healthcare of Atlanta, Atlanta, Ga; ${ }^{\mathrm{d}}$ Department of Pediatric Cardiology, Children's Hospital of Wisconsin, Medical College of Wisconsin, Milwaukee, Wis; ${ }^{\mathrm{e}}$ Ahmanson/UCLA Adult Congenital Heart Disease Center, Los Angeles, Calif; ${ }^{\mathrm{f}}$ Department of Cardiology, Oregon Health and Science University, Portland, Ore; ' Division of Cardiology, Children's National Medical Center, George Washington University School of Medicine, Washington, DC All authors take responsibility for all aspects of the reliability and freedom from bias of the data presented and their discussed interpretation.
}

ventricular volume load. With current techniques, 15-year survival after the Fontan operation approaches $95 \%$. $^{2}$ Long term, however, compromised ventricular preload and increased ventricular afterload and central venous pressures lead invariably to complications that may include

A.M.V. and M.J.L. contributed equally to this work and share senior authorship. Since the completion of this article, C.U. has entered into employment with Novartis. Received for publication Aug 12, 2015; revisions received Sept 19, 2016; accepted for publication Oct 12, 2016; available ahead of print Dec 9, 2016.

Address for reprints: Fred M. Wu, MD, Boston Adult Congenital Heart (BACH) and

Pulmonary Hypertension Program, Boston Children's Hospital, 300 Longwood Ave, Boston, MA 02115 (E-mail: fred.wu@ cardio.chboston.org). $0022-5223 / \$ 36.00$

Copyright (c) 2016 by The American Association for Thoracic Surgery http://dx.doi.org/10.1016/j.jtcvs.2016.10.060 

Abbreviations and Acronyms
AARCC $=$ Alliance for Adult Research in Congenital Cardiology
$\begin{array}{ll}\text { ALT } & =\text { alanine aminotransferase } \\ \text { AST } & =\text { aspartate aminotransferase }\end{array}$
CT = computerized tomography
GGT = gamma glutamyl transpeptidase
HCC = hepatocellular carcinoma
INR = international normalized ratio
IQR = interquartile range
LLN = lower limit of normal
MELD-XI $=$ model for end-stage liver disease, excluding INR
MRI = magnetic resonance imaging
NAFLD $=$ nonalcoholic fatty liver disease
PA $=$ pulmonary artery
PLE = protein-losing enteropathy
RA $=$ right atrium
$\mathrm{RV} \quad=$ right ventricle
ULN = upper limit of normal

Scanning this QR code will take you to the article title page.

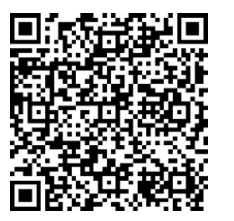

arrhythmias, congestive heart failure, renal insufficiency, plastic bronchitis, protein-losing enteropathy (PLE), and liver disease. ${ }^{3}$

Liver disease has received much attention in recent years as more patients with Fontan circulation enter adulthood and as its impact on long-term morbidity in this population has become apparent. ${ }^{4}$ Increasingly frequent reports of hepatocellular carcinoma (HCC) among patients with Fontan physiology have raised particular concern. ${ }^{5}$ Studies detailing the prevalence of liver disease in patients with Fontan circulation have been restricted to single-center reports consisting of small numbers of patients ${ }^{6-10}$ or with a short period of follow-up. ${ }^{11-13}$ In addition, few have included histopathologic data, which is still considered the "gold standard" for diagnosis of hepatic fibrosis. Biochemical markers of liver disease and hepatic imaging data are more readily available for patients with Fontan circulation but may not accurately reflect the histological changes that are present on biopsy. ${ }^{7-10}$ As such, the prevalence of liver disease and the factors that contribute to more significant liver involvement remain incompletely understood.

To gain better insight into liver health in this population and to form a basis for further prospective studies, the Alliance for Adult Research in Congenital Cardiology
(AARCC) sought to document the prevalence of liver disease and its associated factors in a large, diverse group of adult patients with Fontan physiology. We hypothesized that abnormalities in liver-related serum markers, imaging studies, and histopathology are highly prevalent in this population and are likely to be more pronounced in those with more significant hemodynamic derangement or with more long-standing Fontan circulation.

\section{METHODS}

\section{Study Design}

This was a multi-institutional, cross-sectional, observational study. Subjects were patients 16 years of age or older who had undergone Fontan surgery and who presented to 1 of 6 US adult congenital heart disease (ACHD) centers for evaluation from September 2009 through April 2012. The protocol was developed by the core institution (Boston Children's Hospital, Boston, Mass) and was subsequently refined and approved by members of AARCC. Subjects were excluded if they had a history of cardiac transplantation, inherited forms of liver disease (including hereditary hemochromatosis, Wilson disease, $\alpha 1$-antitrypsin deficiency, and cystic fibrosis) or autoimmune hepatitis. The protocol was approved by each center's institutional review board, and written informed consent was obtained when applicable.

A suggested algorithm for comprehensive assessment of the patient with Fontan circulation, based on established care guidelines and expert consensus, was provided to all centers (Figure 1); however, each subject underwent only those studies deemed clinically indicated by the primary cardiologist. Standardized case report forms were used for data collection, which included basic demographic information, primary congenital cardiac diagnosis as designated using standardized nomenclature, ${ }^{14}$ date of initial Fontan operation, type of initial Fontan operation, other surgical procedures performed, and current medications. Additional data were obtained within 6 months of liver biopsy, or within any 1-year period if no biopsy was performed, including hemodynamics; imaging parameters of cardiac function ${ }^{15}$; cardiopulmonary exercise testing; and laboratory studies, including complete blood count, basic metabolic panel, and liver function tests. For enrolled subjects who had undergone prior liver biopsy, including before 16 years of age, data collected from the 6 months before and after liver biopsy were used. Normal ranges for each center were obtained for use during data analysis.

Hepatic imaging studies included computerized tomography (CT) or magnetic resonance imaging (MRI). These were sent to the core center (Boston Children's Hospital/Brigham and Women's Hospital) in DICOM format where they were reviewed by 2 radiologists (NIS, AJH) who were blinded to all clinical data other than history of Fontan surgery. Cardiac CT and MRI studies, when available, were also reviewed by these radiologists for usable data related to liver health. Available liver biopsies were stained with hematoxylin and eosin, Masson trichrome, and reticulin stain and shipped to the core center for analysis by 2 pathologists (RDO, MEJ), blinded to all clinical data other than history of Fontan surgery. The specimens were graded semiquantitatively for a number of features according to a predetermined scoring system outlined in Table 1, including a scoring scheme for portal fibrosis modified from the METAVIR criteria to provide finer detail in cases of more advanced fibrosis. ${ }^{16}$

\section{Statistical Analysis}

Categorical data are presented as $\mathrm{n}(\%)$ and continuous data are presented as median and range or median and interquartile range as indicated. Characteristics were compared between groups using the Fisher exact test for categorical variables and the Wilcoxon rank sum test for continuous variables. Spearman's rank correlation was used to assess the relationships between ordinal sinusoidal and portal fibrosis scores and 

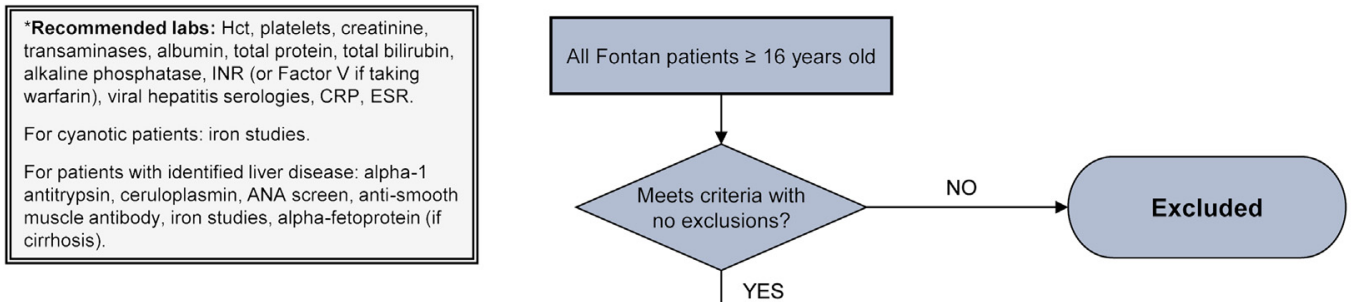
cirrhosis).



FIGURE 1. Suggested algorithm for comprehensive evaluation of subjects. INR, International normalized ratio; $C T$, computerized tomography; $M R$, magnetic resonance.

continuous hemodynamic and laboratory variables. Sinusoidal and portal fibrosis scores were compared by clinical characteristics, imaging findings, and laboratory values based on normal ranges using the Wilcoxon rank sum test. All data analyses were performed with SAS version 9.2 for Windows (SAS Institute, Inc, Cary, NC). Unless otherwise indicated, all tests of significance are 2 -sided with statistical significance judged as $P<.05$.

\section{RESULTS \\ Patient Characteristics}

A total of 241 subjects were enrolled at 6 ACHD centers. Baseline characteristics of the cohort are summarized in Table 2. Compared with previous studies, our cohort was older, with a median age of 25.8 years (range 11.8-59.4 years) and median time since Fontan surgery of 20.3 years (range 5.4-34.5 years). Most had a contemporary-type initial Fontan (53\% lateral tunnel, 2\% extracardiac conduit). The most common primary cardiac diagnoses were tricuspid atresia $(34 \%)$ and double-inlet left ventricle $(24 \%)$. Fontan conversion had been performed in $17 \%$ of subjects, $72 \%$ to an extracardiac connection and $28 \%$ to a lateral tunnel.

The clinical status of subjects varied broadly. The most common complication was arrhythmia, with history of such reported in $61 \%$ of subjects. Moderate or worse ventricular dysfunction was present in $17 \%$ of subjects, and moderate or worse atrioventricular valve regurgitation was present in $15 \%$. Otherwise, reported post-Fontan complications were relatively few.

\section{General Findings}

Excluding a small group of patients who had no liver studies reported $(n=20)$, only $6 \%$ of the remaining cohort ( $n=13$ out of 221) had completely normal assessment of liver health. This group consisted entirely of patients with no radiographic or histologic liver studies.

\section{Laboratory Findings}

At least one laboratory value was available for 217 $(90 \%)$ subjects. Results are summarized in Table 2. In $84 \%$ of patients in whom laboratory studies were performed, at least 1 laboratory value was abnormal. The most commonly and most significantly abnormal was gamma glutamyl transpeptidase (GGT), which was elevated in $75 \%$ of patients (median $69 \mathrm{U} / \mathrm{L}$ [interquartile range (IQR) 46-111]). Thrombocytopenia was present in $41 \%$ of subjects, but most exhibited only mildly depressed platelet counts (median $165 \mathrm{~K}$ cells $/ \mu \mathrm{L}$ [IQR 126-219]). Most patients had normal to slightly elevated transaminases, alkaline phosphatase, and total bilirubin and normal to slightly depressed albumin. 
TABLE 1. Scoring criteria used for histopathological analysis of liver biopsies

\begin{tabular}{|c|c|}
\hline \multicolumn{2}{|c|}{ Sinusoidal fibrosis } \\
\hline 0 & No sinusoidal fibrosis \\
\hline 1 & Sinusoidal fibrosis present in $<1 / 3$ sinusoids \\
\hline 2 & Sinusoidal fibrosis present in $1 / 3-2 / 3$ sinusoids \\
\hline 3 & Sinusoidal fibrosis affecting $>2 / 3$ sinusoids \\
\hline \multicolumn{2}{|c|}{ Portal fibrosis } \\
\hline 0 & No fibrosis \\
\hline 1 & Stellate enlargement of portal tract without septa formation \\
\hline 2 & Enlargement of portal tract with rare septa formation \\
\hline 3 & Numerous septa formation (bridging fibrosis) \\
\hline 4 & Occasional nodules (evolving cirrhosis) \\
\hline 5 & Established cirrhosis \\
\hline \multicolumn{2}{|c|}{ Sinusoidal dilation } \\
\hline 0 & No sinusoidal dilation \\
\hline 1 & Sinusoidal dilation present in $<1 / 3$ sinusoids \\
\hline 2 & Sinusoidal dilation present in $1 / 3-2 / 3$ sinusoids \\
\hline 3 & Sinusoidal dilation affecting $>2 / 3$ sinusoids \\
\hline \multicolumn{2}{|c|}{ Piecemeal necrosis } \\
\hline 0 & Absent \\
\hline 1 & Focal alteration of the periportal plate in some portal tracts \\
\hline 2 & $\begin{array}{l}\text { Diffuse alteration of the periportal plate in some portal } \\
\text { tracts/focal lesion around all portal tracts }\end{array}$ \\
\hline 3 & Diffuse alteration of the periportal plate in all portal tracts \\
\hline \multicolumn{2}{|c|}{ Focal lobular necrosis } \\
\hline 0 & Absent \\
\hline 1 & Less than 1 necroinflammatory foci per lobule \\
\hline 2 & At least 1 necroinflammatory foci per lobule \\
\hline 3 & $\begin{array}{l}\text { Several necroinflammatory foci per lobule or confluent } \\
\text { or bridging necrosis }\end{array}$ \\
\hline \multicolumn{2}{|c|}{ Iron deposition } \\
\hline 0 & Trace \\
\hline 1 & $1+$ \\
\hline 2 & $2+$ \\
\hline 3 & $3+$ \\
\hline \multicolumn{2}{|c|}{ Ductular reaction } \\
\hline 0 & No ductular reaction \\
\hline 1 & $<25 \%$ of portal tracts \\
\hline 2 & $25 \%-75 \%$ of portal tracts \\
\hline 3 & $>75 \%$ portal tracts \\
\hline \multicolumn{2}{|c|}{ Steatosis } \\
\hline 0 & Absent \\
\hline 1 & $<25 \%$ \\
\hline 2 & $25 \%-50 \%$ \\
\hline 3 & $>50 \%$ \\
\hline \multicolumn{2}{|c|}{ Lipofuscin } \\
\hline 0 & Absent \\
\hline 1 & Minimal/focal \\
\hline 2 & $>$ Minimal/focal \\
\hline
\end{tabular}

\section{Imaging Findings}

Hepatic imaging was available for $54(22.4 \%)$ subjects (45 CT, 9 MRI). All studies demonstrated at least 1 abnormal finding, with heterogeneous enhancement of the liver being seen in nearly all cases $(98 \%)$ and an irregular liver contour seen in half (Figure 2). Varices, ascites, and splenomegaly, findings previously reported to be associated with major adverse events in patients with Fontan circulation, were seen in $17 \%, 32 \%$, and $40 \%$ of studies, respectively.

\section{Histopathology Findings}

Liver biopsy was performed in 75 (31.1\%) subjects, 68 of which were judged to be adequate for histopathologic analysis. Of the 68 specimens used for analysis, 54 were obtained via a transvenous approach, 13 were obtained via percutaneous approach, and 1 was obtained at autopsy for a subject who died after enrollment. The original slides for 2 subjects could not be located for analysis by the pathologists. Of the 5 that were inadequate for analysis, all were performed through a transvenous approach. Subjects who underwent biopsy tended to be older (27.9 vs 23.9 years, $P=.02)$ and were more likely to have initially undergone an atriopulmonary ( $47 \%$ vs $32 \%$ ) or atrioventricular $(11 \%$ vs $3 \%)$ Fontan operation, and therefore were more likely to have undergone Fontan conversion. Twenty of the biopsied patients had undergone conversion to a lateral tunnel ${ }^{7}$ or extracardiac connection. ${ }^{13}$ Biopsied patients also were more likely to have an abnormal GGT, albumin, or platelets, and were more likely to have a history of arrhythmia or ascites (Table 2).

Liver biopsy was abnormal in every case, with sinusoidal dilation and sinusoidal fibrosis seen nearly universally (Table 3). Sinusoidal fibrosis was seen in $97 \%$ of cases, with $41 \%$ of subjects falling in the highest stage (stage 3). Portal fibrosis was seen in $90 \%$ of cases; $27 \%$ of cases showed evolving cirrhosis (stage 4) and 6\% showed established cirrhosis (stage 5). Examples of evolving cirrhosis (Figure 3,C) and established cirrhosis (Figure 3, $D)$ are shown in comparison with examples of less pronounced fibrosis (Figure 3, $A$ and $B$ ). Necroinflammatory lesions were less common and were nearly always mild when present. Iron deposition, steatosis and lipofuscin were uncommon and, when present, almost always mild.

\section{Correlations Between Modalities}

In 71 subjects with both laboratory and biopsy data, there was no correlation between number of abnormal laboratory findings and either stage of sinusoidal fibrosis $\left(\mathrm{r}_{\mathrm{s}}=0.21\right.$, $P=.17)$ or stage of portal fibrosis $\left(\mathrm{r}_{\mathrm{s}}=0.20, P=.11\right)$. Individuals with at least 1 abnormal laboratory value had significantly higher median sinusoidal fibrosis stage ( 2 vs $1, P=.007)$ and median portal fibrosis stage (3 vs 1 , $P=.003)$.

For the 23 subjects with both imaging and biopsy data, there was no correlation between number of abnormal imaging findings and either stage of sinusoidal fibrosis $\left(\mathrm{r}_{\mathrm{s}}=0.05, P=.86\right)$ or stage of portal fibrosis $\left(\mathrm{r}_{\mathrm{s}}=-0.09, P=.70\right)$. We found no significant correlation between any specific imaging abnormality and stage of sinusoidal or portal fibrosis. 
TABLE 2. Patient characteristics

\begin{tabular}{|c|c|c|c|c|}
\hline & $\begin{array}{c}\text { Total cohort } \\
(n=241)\end{array}$ & $\begin{array}{l}\text { Subjects without } \\
\text { biopsy }(n=166)\end{array}$ & $\begin{array}{c}\text { Subjects with } \\
\text { biopsy }(n=75)\end{array}$ & $\begin{array}{c}P \text { (biopsy vs } \\
\text { no biopsy) }\end{array}$ \\
\hline Age, y (median, range) & $25.8(11.8-59.4)$ & $23.9(11.8-56.3)$ & $27.9(12.9-59.4)$ & .02 \\
\hline Age at initial Fontan, y (median, range) & $5.0(0.1-40.7)$ & $5.1(0.1-40.7)$ & $4.9(0.6-39.0)$ & NS \\
\hline Time since Fontan, y (median, range) & $20.3(5.4-34.5)$ & $19.5(5.4-32.8)$ & $21.1(11.1-34.5)$ & .02 \\
\hline Male sex, $n(\%)(n=239)$ & $129(54)$ & $93(56)$ & $36(49)$ & NS \\
\hline Congenital heart defect, $\mathrm{n}(\%)$ & & & & NS \\
\hline Tricuspid atresia & $82(34)$ & $51(31)$ & $31(42)$ & \\
\hline Double-inlet left ventricle & $57(24)$ & $45(27)$ & $12(16)$ & \\
\hline Hypoplastic left heart & $17(7)$ & $14(8)$ & $3(4)$ & \\
\hline Other & $85(35)$ & $56(34)$ & $29(39)$ & \\
\hline Initial Fontan type, n (\%) & & & & .01 \\
\hline Lateral tunnel & $127(53)$ & $97(58)$ & $30(40)$ & \\
\hline Extracardiac conduit & $5(2)$ & $5(3)$ & 0 & \\
\hline RA-RV & $13(5)$ & $5(3)$ & $8(11)$ & \\
\hline RA-PA & $88(37)$ & $53(32)$ & $35(47)$ & \\
\hline Other & $8(3)$ & $6(4)$ & $2(3)$ & \\
\hline \multicolumn{5}{|l|}{ Laboratory values } \\
\hline Hematocrit, $\%(n=214)$ & $45(13-68)$ & $46(13-68)$ & $44(27-60)$ & .05 \\
\hline Platelets, $\mathrm{K}$ cells $/ \mu \mathrm{L}(\mathrm{n}=214)$ & $165(29-458)$ & $178(29-458)$ & $152(66-427)$ & .04 \\
\hline AST, U/L (n = 203) & $27(10-159)$ & $27(10-159)$ & $27(10-79)$ & NS \\
\hline ALT, U/L $(\mathrm{n}=206)$ & $26(7-170)$ & $26(8-170)$ & $26(7-81)$ & NS \\
\hline GGT, U/L $(\mathrm{n}=119)$ & $69(5-922)$ & $64(5-282)$ & $84(16-922)$ & .008 \\
\hline Alkaline phosphatase, U/L $(\mathrm{n}=198)$ & $81(7-467)$ & $81(7-443)$ & $80(17-467)$ & NS \\
\hline Total bilirubin, $\mathrm{mg} / \mathrm{dL}(\mathrm{n}=202)$ & $0.8(0.2-4.8)$ & $0.8(0.2-4.8)$ & $0.9(0.3-4.7)$ & .13 \\
\hline Albumin, g/dL $(\mathrm{n}=198)$ & $4.5(1.5-5.5)$ & $4.5(1.5-5.5)$ & $4.2(1.5-5.2)$ & $<.001$ \\
\hline Creatinine, $\mathrm{mg} / \mathrm{dL}(\mathrm{n}=204)$ & $0.8(0.3-2.1)$ & $0.8(0.5-2.1)$ & $0.8(0.3-1.5)$ & NS \\
\hline Hepatitis C status & & & & NS \\
\hline Hepatitis $\mathrm{C}$ antibody positive & $7(5)$ & $3(3)$ & $4(7)$ & \\
\hline Hepatitis $\mathrm{C}$ antibody negative & $136(95)$ & $83(97)$ & $53(93)$ & \\
\hline Unknown & 98 & 80 & 18 & \\
\hline \multicolumn{5}{|l|}{ Medical history reported } \\
\hline Arrhythmia & $147(61)$ & $90(56)$ & $57(78)$ & .001 \\
\hline Protein-losing enteropathy & $12(5)$ & $7(4)$ & $5(7)$ & NS \\
\hline Congestive heart failure & $57(24)$ & $42(26)$ & $15(21)$ & NS \\
\hline Stroke (after Fontan completion) & $24(10)$ & $17(10)$ & $7(10)$ & NS \\
\hline Esophageal varices & $5(2)$ & $2(1)$ & $3(4)$ & .17 \\
\hline Ascites & $42(17)$ & $20(12)$ & $22(30)$ & .002 \\
\hline
\end{tabular}

Measures are presented with $\mathrm{n}=$ number of subjects with available data; if not designated, entire cohort of 241 subjects is reported. $N S$, Not significant; $R A$, right atrium; $R V$, right ventricle; $P A$, pulmonary artery; $A S T$, aspartate aminotransferase; $A L T$, alanine aminotransferase; $G G T$, gamma glutamyl transpeptidase.

Finally, in 54 subjects with both laboratory and imaging data, the median number of abnormal imaging findings was no different between patients with at least 1 abnormal laboratory value and those with no abnormal laboratory values (5 vs $6, P=.32$ ).

\section{Clinical-Histopathological Correlations}

Few laboratory findings were predictive of sinusoidal or portal fibrosis stage. An albumin below the lower limit of normal $(\mathrm{n}=5)$ correlated with a lower sinusoidal fibrosis stage ( 1 vs $2 ; P=.02)$ and with a lower portal fibrosis stage ( 0 vs $3 ; P=.002)$. Only one of the subjects with low albumin was noted to have PLE. A significant relationship was seen between $\mathrm{Hgb} \mathrm{A} 1 \mathrm{c}$ and portal fibrosis stage $\left(\mathrm{r}_{\mathrm{s}}=0.63\right.$; $P=.009)$ but not sinusoidal fibrosis stage $\left(\mathrm{r}_{\mathrm{s}}=0.42\right.$;
$P=.13)$. Hgb A1c measurements were all normal or only mildly increased (range $4.9 \%-6.2 \%$ ), so the clinical significance of this finding is unclear. No correlation was seen between $\mathrm{Hgb} \mathrm{Alc}$ and steatosis or between body mass index and portal fibrosis stage or sinusoidal fibrosis stage.

There was no significant correlation among gender; age; age at initial Fontan; time since initial Fontan; anatomic diagnosis; type of initial Fontan operation; history of palliative operations before Fontan completion; history of Fontan revision; ventricular dysfunction; valvular regurgitation; hemodynamic parameters; or clinical history of clinical complications, such as congestive heart failure, arrhythmia, or ascites, and either sinusoidal fibrosis or portal fibrosis. Subjects who were taking beta-blockers had a slight tendency toward lower portal fibrosis stage 


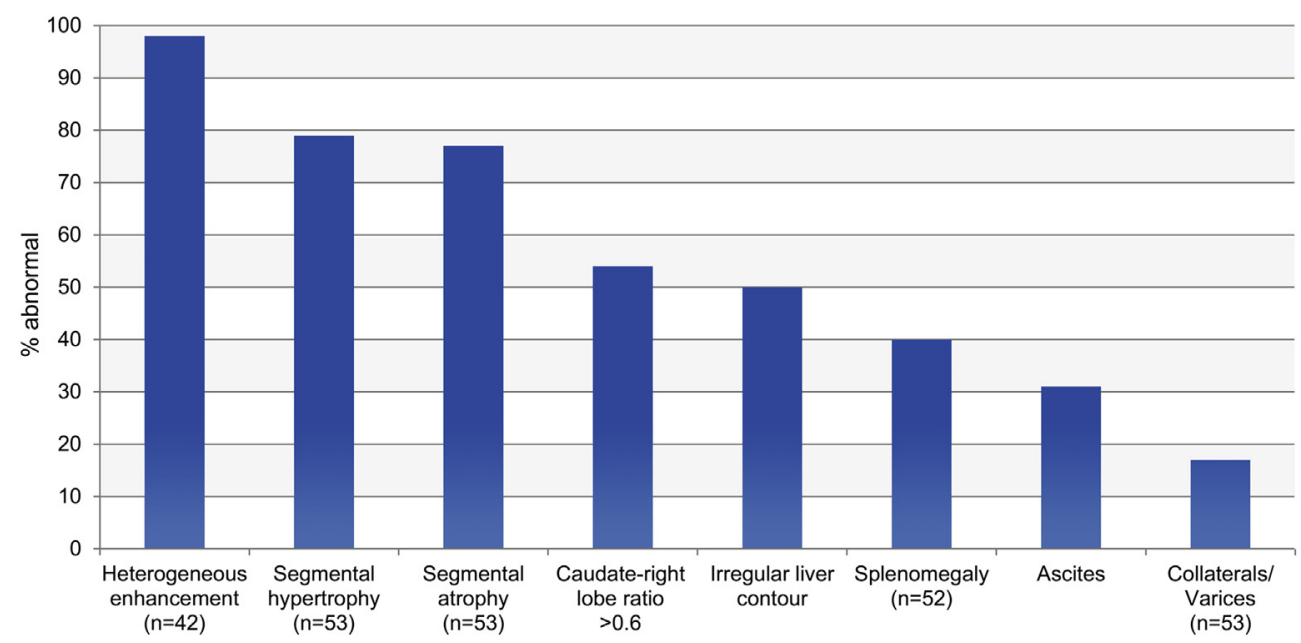

FIGURE 2. Radiologic indicators of liver disease. Due to variations in imaging technique, not all findings could be evaluated in every case. Each finding is presented with $\mathrm{n}=$ number of subjects with available data; if not designated, all 54 imaging studies are reported.

than those who were not $(P=.05)$. No other medications correlated with stage of sinusoidal or portal fibrosis. Among previously identified predictors of poor outcomes, there was no significant correlation between MELD-XI score, ${ }^{17}$ VAST score, ${ }^{18}$ or peak relative $\mathrm{VO} 2^{19}$ and presence of either sinusoidal fibrosis or portal fibrosis on biopsy specimens.

\section{DISCUSSION}

Current evidence suggests that liver disease contributes to earlier mortality in adults after Fontan surgery. ${ }^{17}$ This study is the first multicenter effort to characterize liver health in a large population of adults with Fontan circulation and includes the most representative population to date. Regardless of testing modality, the prevalence of abnormal markers of liver disease among adults with Fontan circulation is remarkably high, with more than $94 \%$ of 221 patients tested having some evidence of liver abnormality. Even among patients with no reported history of post-Fontan complications, the prevalence of liver disease exceeded $86 \%$. The severity of histopathologic disease among the 68 biopsy specimens was notable as well, with $33 \%$ of cases showing regenerative, or cirrhotic, nodules, and $18 \%$ of those, or $6 \%$ of the total, showing established cirrhosis. This is even higher than previously observed in patients with advanced heart failure unrelated to congenital heart disease undergoing evaluation for ventricular assist device or cardiac transplantation. ${ }^{20}$

The recognition that liver disease can be linked to heart and lung disease is not a recent one. Hepatic fibrosis resulting from congestive heart failure has long been described as a primarily sinusoidal process with portal tract involvement in only the most advanced cases. This type of hepatopathy is thought to be related primarily to increased central venous pressures, which, over time, result in hepatic injury and fibrosis due to sinusoidal stasis, stromal stretch, and compression of adjacent hepatocytic plates. ${ }^{21}$ Fibrosis in the portal distribution tends to be associated more with inflammatory liver diseases, such as viral hepatitis, toxic injury related to alcohol or medications, or nonalcoholic fatty liver disease (NAFLD). However,

TABLE 3. Histopathologic findings

\begin{tabular}{|c|c|c|c|c|c|c|c|}
\hline & \multicolumn{6}{|c|}{$\begin{array}{c}\text { Score, } \mathbf{n}(\%) \\
<\text { Less severe more severe }>\end{array}$} & \multirow{2}{*}{$\begin{array}{c}\text { Number abnormal, } \\
\text { n }(\%) \\
\end{array}$} \\
\hline & $\mathbf{0}$ & 1 & 2 & 3 & 4 & 5 & \\
\hline Portal fibrosis $(n=67)$ & $7(10)$ & $11(16)$ & $9(13)$ & $18(27)$ & $18(27)$ & $4(6)$ & $60(90)$ \\
\hline Sinusoidal fibrosis $(\mathrm{n}=63)$ & $2(3)$ & $21(33)$ & $14(22)$ & $26(41)$ & & & $61(97)$ \\
\hline Sinusoidal dilation $(\mathrm{n}=68)$ & $1(1)$ & $24(35)$ & $17(25)$ & $26(38)$ & & & $67(99)$ \\
\hline Piecemeal necrosis $(\mathrm{n}=68)$ & $44(65)$ & $14(21)$ & $10(15)$ & 0 & & & $24(35)$ \\
\hline Focal lobular necrosis $(\mathrm{n}=67)$ & $49(73)$ & $13(19)$ & $4(6)$ & $1(1)$ & & & $18(27)$ \\
\hline Iron deposition $(\mathrm{n}=30)$ & $28(93)$ & $2(7)$ & 0 & 0 & & & $2(7)$ \\
\hline Ductular reaction $(\mathrm{n}=68)$ & $67(99)$ & $1(1)$ & 0 & 0 & & & $1(1)$ \\
\hline Steatosis $(n=68)$ & $55(81)$ & $10(15)$ & $1(1)$ & $2(3)$ & & & $13(19)$ \\
\hline Lipofuscin $(n=68)$ & $60(88)$ & $8(12)$ & 0 & & & & $8(12)$ \\
\hline
\end{tabular}




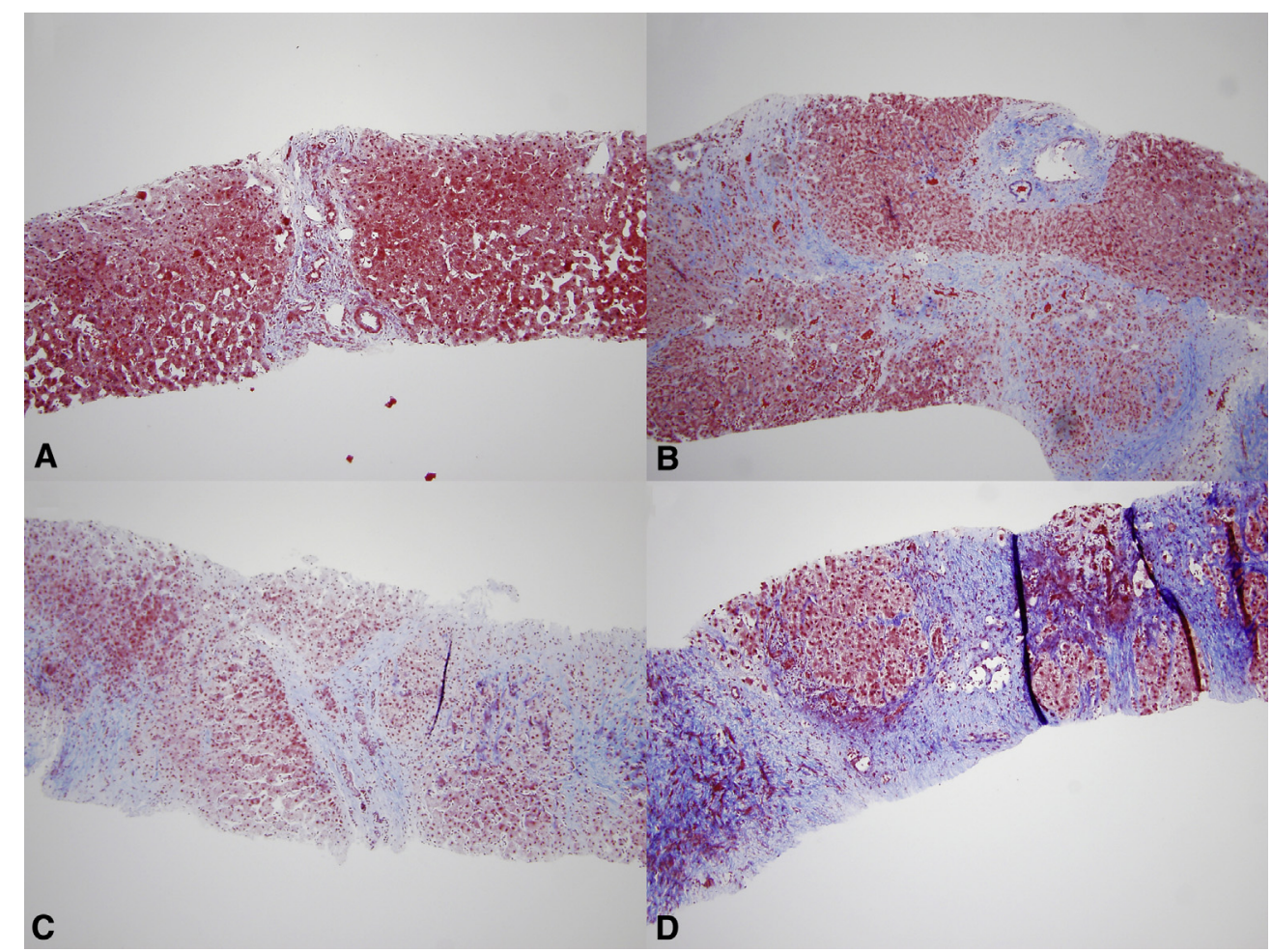

FIGURE 3. A, Mild (stage 1/5) fibrosis surrounding a portal tract. Mild sinusoidal dilation is also seen within the hepatic lobule (Masson trichrome stain). B, More extensive (stage 3/5) fibrosis with broad septa throughout the biopsy core (Masson trichrome stain). C, Advanced (stage 4/5) portal fibrosis with septa formation and evolving regenerative nodule (Masson trichrome stain). D, Thick bands of fibrosis and regenerative nodules consistent with cirrhosis (stage 5/5) (Masson trichrome stain).

consistent with other reports, we find portal involvement in patients with Fontan circulation at all stages of sinusoidal fibrosis, suggesting that factors other than increased central venous pressure may be contributing to their liver injury. ${ }^{7-10}$

Studies of liver disease in Fontan circulation have focused on varying modalities that have included serum biomarkers; measurement of liver stiffness; ultrasound, CT, or MR imaging; biopsy; and autopsy. ${ }^{7-10,12,13,22-33}$ It has remained difficult to draw generalizable conclusions from these studies because correlation between noninvasive testing and severity of histopathologic disease in this population has not been demonstrated. Studies that do focus on histopathology have remained largely retrospective, limited to small numbers, and subject to indication bias (ie, sicker patients are more likely to undergo biopsy).

No correlation between noninvasive testing and severity of fibrosis on liver biopsy was noted in our study. Neither the absolute number of imaging abnormalities nor any individual imaging findings correlated significantly with degree of fibrosis on biopsy. With the exception of a seemingly paradoxical relationship between albumin and fibrosis, no specific biomarker correlated with more advanced hepatic fibrosis. Similarly, no elements of patient clinical history were able to identify those subjects in whom histopathologic evaluation demonstrated more severe fibrosis. No factors previously reported to be related to portal and sinusoidal fibrosis (time since Fontan completion, age at Fontan operation, heterotaxy syndrome, platelet count, and hepatic vein pressure ${ }^{7,8,10}$ ) were found to correlate significantly with severity of either portal or sinusoidal fibrosis in our study.

The correlation between low albumin and lower sinusoidal and portal fibrosis stages was unexpected. It may be that the primary mechanism for liver dysfunction in Fontan physiology is not enhanced extracellular matrix deposition, but rather some degree of direct hepatocyte injury resulting from chronic congestion. As well, a lower serum albumin may be a reflection of poorer nutritional indices in persons with single ventricle physiology or a manifestation of subclinical or undiagnosed PLE rather than a marker of primary liver disease. Increasing $\mathrm{Hgb}$ A1c correlated with more severe portal fibrosis; associations are known to exist between liver disease and insulin resistance, and between diabetes and NAFLD, which is associated with progressive hepatic fibrosis in a primarily portal distribution. ${ }^{34}$ However, Hgb A1c did not appear to correlate with steatosis, as might be expected. This newly detected association warrants further evaluation. 
It is difficult to determine whether the poor correlation among imaging, laboratory, and histologic assessment of liver disease is due to the poor diagnostic yield of the former 2 or to limitations of the latter. Although often considered the "gold standard" for diagnosis, studies in other forms of liver disease have identified shortcomings of liver biopsy, such as interobserver variability and sampling error, particularly in disease processes in which fibrosis is unevenly distributed. ${ }^{35,36}$ This also may explain the poor correlation between histopathology findings and known predictors of major adverse outcomes.

Although it may be easy to find reassurance in the poor correlation between degree of hepatic fibrosis and patient clinical status, the clinical importance of recognizing advanced liver disease in the Fontan population cannot be overstated. ${ }^{17}$ Currently, the Society of Thoracic Surgeons National Database includes at least 800 patients undergoing Fontan operation annually. ${ }^{37}$ If, by 25 years of age, all of these patients will have developed significant liver disease, with $25 \%$ of them having congestive heart failure, the implications for subsequent resource utilization (ie, heart transplant, combined heart-liver transplant, or potentially durable mechanical support at some future time) are staggering. Furthermore, coexisting liver disease in the patient with Fontan circulation has presented a barrier to heart transplantation in many cases. $^{38,39}$ In others, unappreciated liver dysfunction may contribute to increased postoperative mortality. More recently, reports of HCC among older patients with Fontan physiology have been generating particular concern.

With the exception of hepatitis B, HCC rarely arises in the absence of cirrhosis. In cirrhosis, within regenerative nodules, hepatocytes can undergo dysplastic changes that can eventually progress to $\mathrm{HCC}$ at a rate of $1 \%$ to $4 \%$ per year. The estimated 5-year survival rate for $\mathrm{HCC}$ is $9 \%$ and falls to less than $5 \%$ for those who remain untreated. ${ }^{40}$ In this context, the frequency with which regenerative nodules were seen on liver biopsy in our study becomes particularly alarming.

Experts advocate surveillance for $\mathrm{HCC}$ in patients with Fontan circulation who are at risk. Unfortunately, how best to identify those who warrant screening for HCC and the most appropriate screening intervals and modalities are unknown. For this reason, we advocate a proactive approach to surveillance of liver health in the Fontan population that involves some combination of regular laboratory work, hepatic imaging, and liver biopsy when appropriate. However, clinicians must proceed with caution when interpreting such data in patients with Fontan physiology, and collaboration with a hepatologist is strongly recommended. Further studies looking beyond correlations with histopathology and focusing instead on correlations with adverse outcomes, including $\mathrm{HCC}$, are needed to guide our management of this complex patient group.

\section{Limitations}

Our study has important limitations. Liver biopsy was only performed when felt by the managing physicians to be clinically indicated. However, the investigators adopted an aggressive approach to assessment of liver health in this cohort of adults with Fontan circulation, and, in many cases, we performed transvenous liver biopsy for screening purposes at the time of a cardiac catheterization, an electrophysiology study or ablation, or a pacemaker procedure. This is reflected in the large proportion of biopsies that were performed via transjugular approach as opposed to a percutaneous approach $(79.4 \%$ vs $19.1 \%)$. Nevertheless, because not all subjects underwent liver biopsy, our population probably remains somewhat skewed toward sicker patients.

In addition, it remains unclear whether liver biopsy is the most reliable diagnostic test in Fontan liver disease. Heterogeneous distribution of hepatic fibrosis could result in sampling error and underestimation of hepatic fibrosis. Although overestimation of hepatic fibrosis is also a possibility, the observation of regenerative nodules, even if confined to a small region of the liver, may put the patient at risk for developing HCC. As such, these patients likely warrant ongoing surveillance, even if the overall degree of liver disease is mild.

Finally, $41 \%$ of the patients in our cohort had an unknown or unreported hepatitis $\mathrm{C}$ status. The proportion of patients with unknown hepatitis $\mathrm{C}$ status was smaller among those who had undergone liver biopsy, but still approached $25 \%$ in that subgroup. This reflects the patient population likely to be encountered in practice, however, in which hepatitis $\mathrm{C}$ status is often forgotten or never checked despite recommendations to do so. Among those in our study with known hepatitis C status, we found no correlation with degree of sinusoidal or portal fibrosis.

\section{CONCLUSIONS}

Evidence of liver disease is seen almost universally in adults who have undergone Fontan surgery, even in those who would otherwise be considered "well." In as many as $33 \%$ of cases, degree of histological liver disease is severe and may identify a subgroup of patients who are at increased risk for major adverse outcomes, which may include HCC. Few correlations were identified between degree of histopathologic liver disease and patient characteristics or findings on noninvasive testing. Further research is needed to identify the patients at highest risk for significant liver disease. It remains prudent for clinicians caring for adults with Fontan physiology to adopt a surveillance strategy that includes periodic laboratory testing, dedicated hepatic imaging, a low threshold for pursuing tissue diagnostics, and collaborative care with a hepatologist. 


\section{Conflict of Interest Statement}

Authors have nothing to disclose with regard to commercial support.

\section{References}

1. Fontan F, Baudet E. Surgical repair of tricuspid atresia. Thorax. 1971;26:240-8. 2. d'Udekem Y, Iyengar AJ, Cochrane AD, Grigg LE, Ramsay JM, Wheaton GR, et al. The Fontan procedure: contemporary techniques have improved longterm outcomes. Circulation. 2007;116:I157-64.

3. Khairy P, Fernandes SM, Mayer JE Jr, Triedman JK, Walsh EP, Lock JE, et al. Long-term survival, modes of death, and predictors of mortality in patients with Fontan surgery. Circulation. 2008;117:85-92.

4. Wu FM, Ukomadu C, Odze RD, Valente AM, Mayer JE Jr, Earing MG. Liver disease in the patient with Fontan circulation. Congenit Heart Dis. 2011;6:190-201.

5. Asrani SK, Warnes CA, Kamath PS. Hepatocellular carcinoma after the Fontan procedure. N Engl J Med. 2013;368:1756-7.

6. Ghaferi AA, Hutchins GM. Progression of liver pathology in patients undergoing the Fontan procedure: chronic passive congestion, cardiac cirrhosis, hepatic adenoma, and hepatocellular carcinoma. J Thorac Cardiovasc Surg. 2005;129: 1348-52.

7. Johnson JA, Cetta F, Graham RP, Smyrk TC, Driscoll DJ, Phillips SD, et al. Identifying predictors of hepatic disease in patients after the Fontan operation: a postmortem analysis. J Thorac Cardiovasc Surg. 2013;146:140-5.

8. Schwartz MC, Sullivan L, Cohen MS, Russo P, John AS, Guo R, et al. Hepatic pathology may develop before the Fontan operation in children with functional single ventricle: an autopsy study. J Thorac Cardiovasc Surg. 2012;143:904-9.

9. Schwartz MC, Sullivan LM, Glatz AC, Rand E, Russo P, Goldberg DJ, et al. Portal and sinusoidal fibrosis are common on liver biopsy after Fontan surgery. Pediatr Cardiol. 2013;34:135-42.

10. Kiesewetter CH, Sheron N, Vettukattill JJ, Hacking N, Stedman B, MillwardSadler H, et al. Hepatic changes in the failing Fontan circulation. Heart. 2007; 93:579-84.

11. Cromme-Dijkhuis AH, Hess J, Hahlen K, Henkens CM, Bink-Boelkens MT, Eygelaar AA, et al. Specific sequelae after Fontan operation at mid- and longterm follow-up. Arrhythmia, liver dysfunction, and coagulation disorders. J Thorac Cardiovasc Surg. 1993;106:1126-32.

12. Kaulitz R, Luhmer I, Bergmann F, Rodeck B, Hausdorf G. Sequelae after modified Fontan operation: postoperative haemodynamic data and organ function. Heart. 1997;78:154-9.

13. van Nieuwenhuizen RC, Peters M, Lubbers LJ, Trip MD, Tijssen JG, Mulder BJ. Abnormalities in liver function and coagulation profile following the Fontan procedure. Heart. 1999;82:40-6.

14. Franklin RC, Jacobs JP, Krogmann ON, Beland MJ, Aiello VD, Colan SD, et al. Nomenclature for congenital and paediatric cardiac disease: historical perspectives and The International Pediatric and Congenital Cardiac Code. Cardiol Young. 2008;18:70-80.

15. Lang RM, Bierig M, Devereux RB, Flachskampf FA, Foster E, Pellikka PA, et al. Recommendations for chamber quantification: a report from the American Society of Echocardiography's Guidelines and Standards Committee and the Chamber Quantification Writing Group, developed in conjunction with the European Association of Echocardiography, a branch of the European Society of Cardiology. J Am Soc Echocardiogr. 2005;18:1440-63.

16. Intraobserver and interobserver variations in liver biopsy interpretation in patients with chronic hepatitis C. The French METAVIR Cooperative Study Group. Hepatology. 1994;20:15-20.

17. Assenza GE, Graham DA, Landzberg MJ, Valente AM, Singh MN, Bashir A, et al. MELD-XI score and cardiac mortality or transplantation in patients after Fontan surgery. Heart. 2013;99:491-6.

18. Elder RW, McCabe NM, Hebson C, Veledar E, Romero R, Ford RM, et al. Features of portal hypertension are associated with major adverse events in Fontan patients: the VAST study. Int J Cardiol. 2013;168:3764-9.

19. Fernandes SM, Alexander ME, Graham DA, Khairy P, Clair M, Rodriguez E, et al. Exercise testing identifies patients at increased risk for morbidity and mortality following Fontan surgery. Congenit Heart Dis. 2011;6:294-303.

20. Gelow JM, Desai AS, Hochberg CP, Glickman JN, Givertz MM, Fang JC. Clinical predictors of hepatic fibrosis in chronic advanced heart failure. Circ Heart Fail. 2010;3:59-64.
21. Goto T, Mikami KI, Miura K, Ohshima S, Yoneyama K, Nakane K, et al. Me chanical stretch induces matrix metalloproteinase 1 production in human hepatic stellate cells. Pathophysiology. 2004;11:153-8.

22. Baek JS, Bae EJ, Ko JS, Kim GB, Kwon BS, Lee SY, et al. Late hepatic complications after Fontan operation; non-invasive markers of hepatic fibrosis and risk factors. Heart. 2010;96:1750-5.

23. Bryant T, Ahmad Z, Millward-Sadler H, Burney K, Stedman B, Kendall T, et al. Arterialised hepatic nodules in the Fontan circulation: hepatico-cardiac interactions. Int J Cardiol. 2011;151:268-72.

24. Camposilvan S, Milanesi O, Stellin G, Pettenazzo A, Zancan L, D’Antiga L. Liver and cardiac function in the long term after Fontan operation. Ann Thorac Surg. 2008;86:177-82.

25. Chaloupecky V, Svobodova I, Hadacova I, Tomek V, Hucin B, Tlaskal T, et al. Coagulation profile and liver function in 102 patients after total cavopulmonary connection at mid term follow up. Heart. 2005;91:73-9.

26. Friedrich-Rust M, Koch C, Rentzsch A, Sarrazin C, Schwarz P, Herrmann E, et al. Noninvasive assessment of liver fibrosis in patients with Fontan circulation using transient elastography and biochemical fibrosis markers. J Thorac Cardiovasc Surg. 2008;135:560-7.

27. Ginde S, Hohenwalter MD, Foley WD, Sowinski J, Bartz PJ, Venkatapuram S, et al. Noninvasive assessment of liver fibrosis in adult patients following the Fontan procedure. Congenit Heart Dis. 2012;7:235-42.

28. Narkewicz MR, Sondheimer HM, Ziegler JW, Otanni Y, Lorts A, Shaffer EM, et al. Hepatic dysfunction following the Fontan procedure. J Pediatr Gastroenterol Nutr. 2003;36:352-7.

29. Kutty SS, Peng Q, Danford DA, Fletcher SE, Perry D, Talmon GA, et al. Increased hepatic stiffness as consequence of high hepatic afterload in the Fontan circulation: a vascular Doppler and elastography study. Hepatology. 2014;59: 251-60.

30. Wallihan DB, Podberesky DJ. Hepatic pathology after Fontan palliation: spectrum of imaging findings. Pediatr Radiol. 2013;43:330-8.

31. Bulut OP, Romero R, Mahle WT, McConnell M, Braithwaite K, Shehata BM, et al. Magnetic resonance imaging identifies unsuspected liver abnormalities in patients after the Fontan procedure. J Pediatr. 2013;163:201-6.

32. Wu FM, Opotowsky AR, Raza R, Harney S, Ukomadu C, Landzberg MJ, et al. Transient elastography may identify Fontan patients with unfavorable hemodynamics and advanced hepatic fibrosis. Congenit Heart Dis. 2014;9: 438-47.

33. Kutty SS, Zhang M, Danford DA, Hasan R, Duncan KF, Kugler JD, et al. Hepatic stiffness in the bidirectional cavopulmonary circulation: The Liver AdultPediatric-Congenital-Heart-Disease Dysfunction Study group. J Thorac Cardiovasc Surg. 2016;151:678-84.

34. Gupte P, Amarapurkar D, Agal S, Baijal R, Kulshrestha P, Pramanik S, et al. Nonalcoholic steatohepatitis in type 2 diabetes mellitus. J Gastroenterol Hepatol. 2004; 19:854-8.

35. Standish RA, Cholongitas E, Dhillon A, Burroughs AK, Dhillon AP. An appraisal of the histopathological assessment of liver fibrosis. Gut. 2006;55: $569-78$.

36. Wu FM, Jonas MM, Opotowsky AR, Harmon A, Raza R, Ukomadu C, et al. Portal and centrilobular hepatic fibrosis in Fontan circulation and clinical outcomes. J Heart Lung Transplant. 2015;34:883-91.

37. The Society of Thoracic Surgeons. STS congenital heart surgery executive summary, all patients, Jan 2012-Dec 2015. Chicago (IL): The Society of Thoracic Surgeons; June 28, 2016. Available at: http://www.sts.org/sites/ default/files/documents/Congenital_STSExecSummary_AllPatients6.28.16.pdf. Accessed September 16, 2016.

38. Haller C. Taking care all along the way. J Thorac Cardiovasc Surg. 2016;151: 685-6.

39. Jaquiss RD. Hypoplastic left heart syndrome: how to improve late survival and quality of life. J Thorac Cardiovasc Surg. 2015;150:1401-3.

40. Ries LAG, Young JL, Keel GE, Eisner MP, Lin YD, Horner M-J. SEER Survival Monograph: Cancer Survival Among Adults: U.S. SEER Program, 1988-2001, Patient and Tumor Characteristics. Bethesda, MD: National Cancer Institute, SEER Program; 2007. NIH Pub. No. 07-6215.

Key Words: Fontan circulation, congestive hepatopathy, hepatic fibrosis, congenital heart disease, adult congenital heart disease 